\title{
Determinación de la carga al dorso en caballos de rodeo chileno y comparación con la capacidad máxima de carga al dorso estimada, de acuerdo a la fórmula de Beltrán (1954)
}

\author{
Determination of the back load in horses used for Chilean rodeo and comparison with \\ the estimated back load maximum capacity, in accordance with the Beltrán formula (1954)

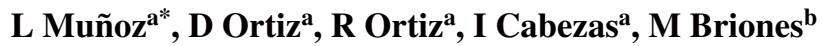 \\ aDepartamento de Ciencias Clínicas, Facultad de Ciencias Veterinarias, Universidad de Concepción, Chillán, Chile. \\ bepartamento de Ciencias Pecuarias, Facultad de Ciencias Veterinarias, Universidad de Concepción, Chillán, Chile.
}

\begin{abstract}
SUMMARY
This study was aimed to determine the weight supported on the back of the Chilean horse during the Chilean rodeo and to determine if this weight represents an overload for the animal, according to the Beltrán formula (1954). One hundred and forty registered Chilean horses, 74 geldings, 42 mares and 24 stallions (ages from 5 to 22 years) participants in rodeos at the O'Higgins Region were sampled. The height to the withers and girth in each individual was obtained in order to establish the back load maximum capacity. Riders and saddles were weighed to calculate the actual load supported by each horse and the actual percentage used of the maximun load was estimated. The results obtained indicate that Chilean rodeo horses present average height to the withers and average girth within the breed's standard. Among the horses assessed $32.15 \%$ had a height to the withers below the lower limit of the established breed standard, and $6.43 \%$ showed girth size below the standard. The actual back load obtained was in average of $93.4 \pm 11.22$ $\mathrm{kg}$, being the rider's weight the main component of the total load. The estimated back load maximum capacity was in average $115.85 \pm 6.07 \mathrm{~kg}$. The relationship between the estimated back load maximum capacity and the actual back load showed that $3.57 \%$ of the horses assessed were overloaded, with an overall average for this ratio $80.78 \pm 10.02 \%$.
\end{abstract}

Palabras clave: equinos, Caballo Chileno, alzada, perímetro torácico, carga al dorso.

Key words: equine, Chilean horse, height to the withers, girth, back load.

\section{INTRODUCCIÓN}

El principal uso del Caballo Chileno es el rodeo chileno (García y col 1997), deporte que se realiza dentro de un picadero circular con superficie de arena (Federación de Rodeo Chileno 2008), en donde un collera formada por dos caballos y sus respectivos jinetes deben arrear al galope y luego detener un novillo de más de $300 \mathrm{~kg}$, presionándolo contra una superficie acolchada denominada quincha, recorriendo aproximadamente $400 \mathrm{~m}$ (García y col 1997) a una velocidad de $6,95 \pm 1,17 \mathrm{~m} / \mathrm{s}$ (Pérez y col 1997). El caballo debe soportar en su dorso el peso del jinete y los aperos correspondientes, que representan aproximadamente el 24\% del peso del caballo (Pérez y col 1997). Según Rooney (1978), la carga al dorso afecta directamente el centro de gravedad e implica una sobrecarga del peso que deben soportar los cuatro miembros, pero sobre todo los miembros anteriores.

Para estimar la capacidad máxima de carga al dorso, Beltrán (1954) propuso una fórmula en que el perímetro torácico al cuadrado dividido por la alzada es multiplicado por un coeficiente que varía dependiendo de si la aptitud

Aceptado: 19.07.2012.

* Casilla 160-C correo 3, Concepción, Chile; lismunoz@udec.cl del caballo es de velocidad, fuerza o mixta. Al respecto, se han realizado sólo dos estudios en el Caballo Chileno, los que concluyeron que el $100 \%$ de los caballos soportan una sobrecarga al dorso durante el rodeo chileno de entre 27 y $36 \%$ (García y col 1997, Moreno 2004). Sin embargo, en ambos estudios, los autores erróneamente usaron el coeficiente para caballo con aptitud para velocidad (coeficiente 35), cuando el caballo se desplaza al galope durante el rodeo chileno y el coeficiente para caballo con aptitud mixta (coeficiente 56) cuando el caballo de desplazaba al paso, por lo que sus resultados y conclusiones respecto a la carga que soporta al dorso el Caballo Chileno durante el rodeo chileno son erróneas.

Con estos antecedentes, el objetivo de nuestro estudio fue determinar la carga al dorso que soporta un Caballo Chileno durante el rodeo, establecer si este peso representa realmente una sobrecarga para el caballo, en relación a la capacidad máxima de carga al dorso estimada en base a la fórmula propuesta por Beltrán (1954) para equinos con aptitud mixta (coeficiente de 56).

\section{MATERIAL Y MÉTODOS}

Se evaluaron 140 Caballos Chilenos inscritos de entre 5 y 22 años y sus respectivos jinetes, los cuales fueron elegidos al azar, todos participantes en rodeos de las Asociaciones de 
Rodeo Chileno Cachapoal y Cardenal Caro, de la Región de O'Higgins, entre los meses de marzo y mayo de 2011.

Para medir la alzada se utilizó el método descrito por Hickman y Colles (1984), para lo cual un cartabón (pie de metro) de $1,8 \mathrm{~m}$, graduado cada $0,5 \mathrm{~cm}$ (marca Hauptner®), se apoyó en el suelo paralelo al miembro anterior izquierdo y se registró la altura del ápice de la quinta vértebra torácica. Para medir el perímetro torácico se utilizó el método descrito por Ellis y Hollands (1998), para lo cual una cinta métrica graduada cada $0,1 \mathrm{~cm}$ (marca Stanley®) se pasó por detrás de la cruz (ápice de la sexta vértebra torácica), adosada al tórax y por detrás de los codos, completando la circunferencia hasta volver al punto de inicio, una vez finalizada la fase de espiración respiratoria. Para el registro del peso de los jinetes con su vestuario reglamentario (sombrero, camisa, chaqueta de huaso, manta o chamanto, pantalón, cinturón, flequillo, polainas, zapatos huasos, taloneras y espuelas) se utilizó una balanza portátil para $130 \mathrm{~kg}$ (marca Ecolé®) sobre una superficie dura, lisa y horizontal. El mismo sistema de utilizó para pesar los aperos que van sobre el dorso (pelero, montura, lazo de cuero, cinchón, dos cinchas, malcornera, aciones y estribos de madera).

En una ficha de registro se anotaron el nombre de cada caballo, sexo (hembra, macho entero o macho castrado), edad, perímetro torácico y alzada en metros, también se incluyeron en ésta el peso del jinete y aperos en kilos. Una vez obtenidos estos antecedentes se sumaron el peso del jinete y aperos, obteniéndose la carga real al dorso de cada caballo. Posteriormente, utilizando la alzada y perímetro torácico, se estimó la capacidad máxima de carga al dorso de acuerdo a la fórmula propuesta por Beltrán (1954) para equinos con aptitud mixta:

Capacidad máxima de carga al dorso $=\frac{\left(56 * P T^{2}\right)}{A}$

donde: PT: perímetro torácico.

A: alzada.

56: coeficiente, para equinos con aptitud mixta de acuerdo a la actividad que realiza (Porte 1978).

La relación entre la capacidad de carga real y la capacidad máxima de carga al dorso estimada, se calculó porcentualmente dividiendo la carga real por la capacidad máxima de carga al dorso estimada.

\section{ANÁLISIS ESTADÍSTICO}

Se determinaron estadígrafos de tendencia central y de dispersión para las variables descritas, mediante la planilla MS Excel de Microsoft $®$.

\section{RESULTADOS Y DISCUSIÓN}

Se evaluaron $74(52,86 \%)$ machos castrados, 42 (30\%) hembras y $24(17,14 \%)$ machos enteros.

En el cuadro 1 se muestran el promedio, desviación estándar y rango de la alzada y perímetro torácico de los Caballos Chilenos, de acuerdo al sexo.

En relación a la alzada, no se observan diferencias entre los tres grupos, sin embargo, aun cuando el promedio de alzada está dentro del rango fijado por el estándar oficial de la Asociación de Criadores de Caballares Chilenos (1978), es bajo principalmente para los machos (enteros y castrados) al compararlo con el promedio ideal de 1,42 m (Porte 2000). Llama la atención que sólo el 67,85\% de los equinos evaluados se encuentran dentro del rango fijado por el estándar oficial de la raza que es de 1,38 - 1,48 m para machos ( $\sin$ hacer diferencias entre enteros y castrados) y 1,36 - 1,46 m para hembras (Asociación de Criadores de Caballares Chilenos 1978), lo cual ya había sido reportado en otros estudios (Valdivia 1995, Ubilla 1996, García y col 1997, Turu 2000, Moreno 2004). Sin embargo, todos los caballos que están fuera del estándar es porque su alzada es menor al límite inferior a diferencia de lo reportado por García y col (1997) y Moreno (2004), quienes reportaron ejemplares con alzada superior al estándar. Al analizar la variable alzada de acuerdo al sexo encontramos que un $28,57 \%$ de las hembras, $41,67 \%$ de los machos enteros y $31,08 \%$ de los machos castrados tienen una alzada menor al estándar, siendo estos porcentajes menores en los machos que los reportados por García y col (1997) y por Moreno (2004), quienes encontraron un 50\% de machos enteros con alzada menor al estándar. La baja alzada registrada, sobre todo en los machos castrados, al parecer es una tendencia como señalan otros autores (Herrera 1979, García

Cuadro 1. Promedio, desviación estándar (DE) y rango de la alzada y perímetro torácico de Caballos Chilenos, de acuerdo al sexo. Average, standard deviation (SD) and range for height of the withers and girth in Chilean horses by sex.

\begin{tabular}{|c|c|c|c|c|c|}
\hline \multirow{2}{*}{ Sexo } & \multirow{2}{*}{$\mathrm{N}^{\mathrm{o}}$ animales } & \multicolumn{2}{|c|}{ Alzada (m) } & \multicolumn{2}{|c|}{ Perímetro torácico $(\mathrm{m})$} \\
\hline & & Promedio $\pm \mathrm{DE}$ & Rango & Promedio $\pm \mathrm{DE}$ & Rango \\
\hline Hembras & 42 & $1,39 \pm 0,03$ & $1,32-1,45$ & $1,70 \pm 0,05$ & $1,60-1,84$ \\
\hline Machos castrados & 74 & $1,39 \pm 0,03$ & $1,31-1,47$ & $1,69 \pm 0,05$ & $1,56-1,80$ \\
\hline Machos enteros & 24 & $1,39 \pm 0,04$ & $1,31-1,46$ & $1,69 \pm 0,04$ & $1,60-1,76$ \\
\hline Total & 140 & $1,39 \pm 0,03$ & $1,31-1,47$ & $1,69 \pm 0,05$ & $1,56-1,84$ \\
\hline
\end{tabular}


y col 1997), que podría ser motivada según Araya (1980), por el hecho de que al darle a un caballo mayor luz desde el suelo, éste alarga sus miembros, cambia su centro de gravedad y desestabiliza el conjunto, restándole fuerza y seguridad a sus movimientos, cualidades necesarias para que el caballo durante la atajada detenga al novillo en un punto preciso y a gran velocidad.

En relación al perímetro torácico, el $93,57 \%$ de los caballos estaban dentro del rango establecido en el estándar de la raza, el cual varía entre 1,62 y 1,82 m en machos y 1,64 y 1,84 m en hembras (Asociación de Criadores de Caballares Chilenos 1978), datos levemente inferiores a los obtenidos por Moreno (2004), quien reportó que un 97\% de los equinos cumplían con el estándar. Los promedios encontrados en hembras, machos enteros y castrados son similares, aunque levemente menores a los obtenidos por Turu (2000) y Moreno (2004), pero esta diferencia es mayor al compararla con estudios anteriores (Valdivia 1995, Ubilla 1996). Tal vez debido a que actualmente existe una tendencia a correr los caballos con una condición corporal menor. Las hembras en general presentaron un perímetro torácico mayor que los machos castrados y enteros, similar a lo reportado por otros autores (Herrera 1979, Pinochet 1980, García y col 1997), lo que era esperable ya que el estándar le asigna $2 \mathrm{~cm}$ más al perímetro torácico de las hembras (Asociación de Criadores de Caballares Chilenos 1978) por razones de tipo reproductivo (Porte 2000). De los equinos fuera del estándar, $9(6,43 \%)$ tenían un perímetro torácico menor al estándar (4 machos castrados,
4 hembras y 1 macho entero), lo que representa más del doble de lo encontrado por Moreno (2004), quien reportó un 3\% bajo el estándar.

En el cuadro 2 se muestran el promedio, desviación estándar y rango de la capacidad máxima de carga al dorso estimada de los Caballos Chilenos evaluados de acuerdo al sexo. En relación a la alzada y perímetro torácico registrados, el mayor valor se obtuvo en las hembras, seguido por los machos castrados y los machos enteros. Estos resultados eran esperables y coinciden con lo reportado por García y col (1997) y Moreno (2004).

En el cuadro 3 se muestran el promedio, desviación estándar y rango de peso de los jinetes y aperos de Caballos Chilenos, de acuerdo al sexo. En relación al peso del jinete con su traje tradicional, un $45,71 \%$ pesaba más de $80 \mathrm{~kg}$, similar a lo reportado por García y col (1997) y Moreno (2004), de 42,2\% y 57\% respectivamente. Sólo un 5\% pesaba más de $100 \mathrm{~kg}$, resultado muy inferior a los reportados por García y col (1997) y Moreno (2004), quienes encontraron porcentajes de $11,5 \%$ y $10 \%$, respectivamente, lo que podría considerarse como una tendencia, en que se busca disminuir el peso al dorso y esfuerzo del caballo. En relación al sexo fue en los machos castrados en donde se encontró jinetes de mayor peso, $36(48,65 \%)$ con peso mayor a 80 kilos, de los cuales $7(9,46 \%)$ pesaban más de 100 kilos, resultado similar al obtenido por García y col (1997). En las hembras se encontraron $19(45,24 \%)$ jinetes con peso mayor a 80 kilos y en los machos enteros sólo 9 $(37,5 \%)$. Sin embargo, no parece haber relación entre el

Cuadro 2. Promedio, desviación estándar (DE) y rango de la capacidad máxima de carga al dorso estimada en Caballos Chilenos, de acuerdo al sexo.

Average, standard deviation and range for estimated back load maximum capacity in Chilean horses by sex.

\begin{tabular}{lccc}
\hline \multirow{2}{*}{ Sexo } & \multirow{2}{*}{$\mathrm{N}^{\mathbf{o}}$ animales } & \multicolumn{2}{c}{ Capacidad máxima de carga al dorso estimada (kg) } \\
\cline { 3 - 4 } & & Promedio $\pm \mathrm{DE}$ & Rango \\
\hline Hembras & 42 & $116,62 \pm 6,31$ & $106,19-136,4$ \\
Machos castrados & 74 & $115,60 \pm 6,40$ & $99,86-131,43$ \\
Machos enteros & 24 & $115,25 \pm 4,44$ & $106,99-122,16$ \\
\hline Total & 140 & $115,85 \pm 6,07$ & $99,86-136,4$ \\
\hline
\end{tabular}

Cuadro 3. Promedio, desviación estándar (DE) y rango, de los pesos de los jinetes y aperos de Caballos Chilenos, de acuerdo al sexo. Average, standard deviation and range for rider plus saddle weights of Chilean horses by sex.

\begin{tabular}{|c|c|c|c|c|c|}
\hline \multirow{2}{*}{ Sexo } & \multirow{2}{*}{$\mathrm{N}^{\circ}$ animales } & \multicolumn{2}{|c|}{ Peso jinetes (kg) } & \multicolumn{2}{|c|}{ Peso aperos (kg) } \\
\hline & & Promedio $\pm \mathrm{DE}$ & Rango & Promedio $\pm \mathrm{DE}$ & Rango \\
\hline Hembras & 42 & $79,62 \pm 8,09$ & $60-100$ & $11,17 \pm 2,39$ & $8-15$ \\
\hline Machos castrados & 74 & $83,15 \pm 11,83$ & $58-115$ & $11,96 \pm 2,4$ & $8-16$ \\
\hline Machos enteros & 24 & $81,33 \pm 10,1$ & $65-100$ & $11,33 \pm 2,57$ & $8-20$ \\
\hline Total & 140 & $80,78 \pm 10,02$ & $58-115$ & $11,62 \pm 2,3$ & $8-20$ \\
\hline
\end{tabular}


Cuadro 4. Promedio, desviación estándar (DE) y rango de carga real al dorso y del porcentaje ocupado de la capacidad máxima de carga al dorso estimada en Caballos Chilenos, de acuerdo al sexo. horses by sex

Average, standard deviation and range for actual back load and percentage of used estimated back load maximum capacity in Chilean

\begin{tabular}{|c|c|c|c|c|c|}
\hline \multirow{2}{*}{ Sexo } & \multirow{2}{*}{$\mathrm{N}^{\circ}$ animales } & \multicolumn{2}{|c|}{ Carga real al dorso $(\mathrm{kg})$} & \multicolumn{2}{|c|}{$\begin{array}{l}\text { Porcentaje ocupado de la capacidad } \\
\text { máxima de carga al dorso estimada }\end{array}$} \\
\hline & & Promedio $\pm \mathrm{DE}$ & Rango & Promedio $\pm \mathrm{DE}$ & Rango \\
\hline Hembras & 42 & $90,79 \pm 8,82$ & $70-112$ & $78,02 \pm 8,22$ & $62,99-92,99$ \\
\hline Machos castrados & 74 & $95,11 \pm 12,33$ & $66-130$ & $82,41 \pm 10,66$ & $54,88-112,73$ \\
\hline Machos enteros & 24 & $92,67 \pm 10,8$ & $77-116$ & $80,55 \pm 10,18$ & $65,10-104,74$ \\
\hline Total & 140 & $93,40 \pm 11,22$ & $66-130$ & $80,78 \pm 10,02$ & $54,88-112,73$ \\
\hline
\end{tabular}

peso del jinete y la elección del sexo del caballo. En relación al peso de los aperos, no se encontraron diferencias entre los grupos, ya que en general pocos aperos pesaban $15 \mathrm{~kg}$ o más, práctica que busca disminuir el peso al dorso y esfuerzo del caballo durante la competencia.

En el cuadro 4 se muestran el promedio, desviación estándar y rango de carga real al dorso y del porcentaje ocupado de la capacidad máxima de carga al dorso estimada en Caballos Chilenos, de acuerdo al sexo. En relación a la carga real al dorso, considerando como carga total el peso del jinete y sus aperos, los machos castrados presentaron una mayor carga real al dorso, seguido por los machos enteros y por último las hembras, resultados similares a los reportados por García y col (1997). Esto se debe a la mayor variabilidad en el peso de los jinetes del grupo de machos castrados y a que en ese grupo se encontraron las monturas de mayor peso. En la relación al porcentaje ocupado por la carga real al dorso en relación a la capacidad máxima de carga al dorso estimada, fue en los machos castrados en donde se encontraron los porcentajes mínimos y máximos. Los equinos evaluados en este estudio poseían un promedio de carga real al dorso de $93,40 \mathrm{~kg}$ ocupando un $80,78 \%$ de la capacidad máxima de carga al dorso estimada según la fórmula propuesta por Beltrán (1954), porcentaje levemente menor (2\%) al que estimaron García y col (1997) y Moreno (2004) en Caballos Chilenos al paso, quienes reportaron $82,87 \%$ y $82,7 \%$, respectivamente, pero a su vez inferior a los reportados por estos mismos autores para caballos al galope (compitiendo), ya que ambos concluyeron que el $100 \%$ de los Caballos Chilenos en competencia soportaban una sobrecarga al dorso de 27 y $36 \%$, respectivamente. Esta diferencia se debe a que en ambos estudios los autores aplicaron a la fórmula de Beltrán un coeficiente diferente si el caballo se desplazaba al paso o al galope, en circunstancias que el coeficiente se aplica de acuerdo a la aptitud del caballo y no al aire en que se desplaza. En el presente estudio, sólo $5(3,57 \%)$ caballos soportaba una carga al dorso mayor a la capacidad máxima de carga al dorso estimada de acuerdo a sus medidas hipométricas, de los cuales 4 eran machos castrados y 1 macho entero.
Nuestro trabajo permite concluir que la carga al dorso que soporta un Caballo Chileno durante el rodeo chileno, en general, no representa una sobrecarga para el caballo, en relación a la capacidad máxima de carga al dorso estimada, lo que contrasta con lo reportado previamente por García y col (1997) y Moreno (2004).

\section{RESUMEN}

Los objetivos de este estudio fueron determinar la carga al dorso que soporta un caballo chileno durante el desarrollo del rodeo chileno y si este peso representa una sobrecarga para el caballo, en relación a la capacidad máxima estimada en base a la fórmula propuesta por Beltrán (1954). Se evaluaron 140 Caballos Chilenos inscritos, 74 machos castrados, 42 hembras y 24 machos enteros, de entre 5 y 22 años, participantes en rodeos de la Región de O’Higgins. En cada caballo se midió la alzada y perímetro torácico, para estimar la capacidad máxima de carga al dorso según la fórmula propuesta por Beltrán. Además, se pesó a cada jinete y sus respectivos aperos para calcular la carga real al dorso que soportó cada caballo. Finalmente, se determinó el porcentaje ocupado de la capacidad máxima de carga al dorso estimada en los animales. En relación a las medidas hipométricas, los resultados indican que los caballos de rodeo chileno evaluados presentaron en su mayoría promedios de alzada y perímetro torácico que se encontraban dentro del estándar establecido para la raza, aunque un $32,15 \%$ presentó alzada por debajo del límite inferior del rango establecido y un 6,43\% del total presentó un perímetro torácico por debajo del rango propuesto en el estándar. La carga real al dorso promedio fue $93,40 \pm 11,22 \mathrm{~kg}$, siendo el peso del jinete la principal variable de la carga total. La capacidad máxima de carga al dorso estimada promedio fue 115,85 $\pm 6,07 \mathrm{~kg}$. En relación al porcentaje ocupado de la capacidad máxima de carga al dorso estimada, el promedio general para esta relación fue de 80,78 $\pm 10,02 \%$, sin embargo, se encontró que un $3,57 \%$ de los caballos soportaban un peso al dorso superior al máximo estimado. Se concluye que el peso al dorso que soporta un Caballo Chileno durante el rodeo, en general, no representa una sobrecarga para el caballo en relación a la capacidad máxima de carga al dorso estimada.

\section{REFERENCIAS}

Araya A. 1980. El caballo criollo chileno: origen e historia. Ayer y hoy. Campesino (Chile) 110, 42-59.

Asociación de Criadores de Caballares Chilenos. 1978. Standard del caballo chileno. Rev Fed Rodeo Chileno Asoc Criadores Caballos Chilenos 30, 13-14.

Beltrán J. 1954. Ganado caballar. Salvat SA, Barcelona, España. 
Ellis JM, T Hollands. 1998. Accuracy of different methods of estimating the weight of horses. Vet Rec 143, 335-336.

Federación de Rodeo Chileno. 2008. Medialuna, única, grande y nuestra. Anuario Rodeo Chileno 2008 60, 29-31.

García M, I Cabezas, R Guzmán, S Valenzuela, V Merino, R Pérez. 1997. Características hipométricas, peso corporal y capacidad de carga del caballo fina sangre chileno en rodeo. Av Cien Vet 12, 45-51.

Herrera A. 1979. Valores hipométricos de la raza chilena en la provincia de Nuble. Memoria de título, Facultad de Ciencias Agropecuarias y Forestales, Universidad de Concepción, Chillán, Chile.

Hickman J, C Colles. 1984. Measurement of horses. Vet Rec 144, 491-493.

Moreno R. 2004. Capacidad de carga al dorso del caballo chileno y su relación con la carga real a desplazar en competencias oficiales de la Asociación de Rodeo Chileno de Malleco. Tesis Licenciatura, Escuela de Medicina Veterinaria, Universidad Católica de Temuco, Temuco, Chile.

Pérez R, M García, I Cabezas, R Guzmán, V Merino, S Valenzuela, C González. 1997. Actividad física y cambios cardiovasculares y bioquímicos del caballo chileno a la competencia del rodeo. Arch Med Vet 29, 221-234.
Pinochet JL. 1980. Estudio hipométrico y morfológico del caballo de raza criolla chilena y su posible cambio tipológico. Memoria de título, Escuela de Medicina Veterinaria, Universidad de Chile, Santiago, Chile.

Porte E. 1978. El nuevo estándar del caballo chileno. Rev Fed Rodeo Chileno Asoc Criadores Caballos Chilenos 30, 16-20.

Porte E. 2000. Crecimiento y desarrollo del caballo criollo chileno. Av Prod Anim 25, 167-174.

Rooney J. 1978. Force exerted on the horse's legs. J Equine Med Surg 2, 49.

Turu M. 2000. Estudio hipométrico y conformacional del caballo criollo chileno de la Décima Región. Memoria de título, Facultad de Medicina Veterinaria, Universidad de Concepción, Chillán, Chile.

Ubilla C. 1996. Estudio hipométrico y morfológico del caballo fina sangre criollo chileno en la Octava Región. Memoria de título, Facultad de Medicina Veterinaria, Universidad de Concepción, Chillán, Chile.

Valdivia V. 1995. Estudio hipométrico y conformacional del caballo fina sangre chileno de la Cuarta Región. Memoria de título, Facultad de Medicina Veterinaria, Universidad de Concepción, Chillán, Chile. 